\title{
Multiple Window Time-Frequency Distribution and Coherence of EEG Using Slepian Sequences and Hermite Functions
}

\author{
Yan $\mathrm{Xu}$, Simon Haykin,* Fellow, IEEE, and Ronald J. Racine
}

\begin{abstract}
Multiple window (MW) time-frequency analysis (TFA) is a newly developed technique to estimate a time-varying spectrum for random nonstationary signals with low bias and variance. In this paper, we describe the application of MW-TFA techniques to electroencephalogram (EEG) and compare the results with those of the conventional spectrogram. We find that the MW-TFA provide us with not only low bias and variance time-frequency (TF) distribution for EEG but also TF coherence estimation between a single realization of EEG recorded from two sites. We also compare the performance of the MW-TFA using two sets of windows, Slepian sequences, and Hermite functions. If care is taken in matching the two windows, we find no noticeable difference in the resulting TF representations.
\end{abstract}

Index Terms - Electroencephalogram (EEG), Hermite functions, multiple window (MW), Slepian sequences, spectrogram, time-frequency analysis (TFA).

\section{INTRODUCTION}

$\mathbf{E}$ LECTROENCEPHALOGRAM (EEG) is the summed electrical activity of very large numbers of neurons [1]. It can be recorded from scalp electrodes in human subjects or from electrodes implanted in specific brain regions of experimental animals. EEG recordings have been used extensively to monitor neuronal activation patterns and the flow of neuronal signals between brain sites [2]-[4].

The EEG is a nonstationary signal [5]. The time-frequency (TF) structure of EEG signal is usually represented by conventional TF methods such as the spectrogram wavelet transform (WT) [6], the Wigner-Ville distribution (WVD), or the reduced interference distribution (RID) [7]. These conventional methods are designed for deterministic signals. The EEG, however, is never fully deterministic. Hence, it is usually treated as a random or stochastic process. Research in TFA, to date, has been focused on deterministic signals. Only recently has attention turned to nonstationary random processes [8]-[10].

Manuscript received November 11, 1997; revised January 8, 1999. Asterisk indicates corresponding author.

$\mathrm{Y}$. $\mathrm{Xu}$ is with the Communications Research Laboratory, McMaster University, Hamilton, Ontario L8S 4K1, Canada. She is also with the Department of Psychology, McMaster University, Hamilton, Ont. L8S 4K1, Canada.

*S. Haykin is with the Communications Research Laboratory, McMaster University, Hamilton, Ont L8S 4K1, Canada (e-mail: haykin@synapse.crl.mcmaster.ca).

R. J. Racine is with the Department of Psychology, McMaster University, Hamilton, Ont. L8S 4K1, Canada.

Publisher Item Identifier S 0018-9294(99)04701-1.
To design a TF distribution estimator for a random process, we must consider the bias/variance dilemma. Recently several researchers have extended Thomson's multiple-window method (MWM) to random nonstationary signals to construct a low bias and variance time-varying spectral estimator [9], [10].

In this paper, we apply the multiple-window (MW) timefrequency analysis (TFA) to EEG signals recorded from the entorhinal cortex and hippocampus of the rat. The hippocampus is a brain structure involved in spatial memory and the integration of sensory information. Much of the information the hippocampus receives from cortical areas, including the olfactory cortex, is funneled through the entorhinal cortex. The objective in this research is to determine the frequencies for optimal signal transmission from the olfactory cortex to the hippocampus via the entorhinal cortex.

In this paper, we do the following.

- Describe the application of the MW-TFA to EEG signals.

- Describe the construction of a MW TF coherence function to study the temporal relationship between EEG signals in different regions of the brain.

- Compare the performance of the MW-TFA using two sets of windows, Slepian sequences, and Hermite functions.

- Finally, we report on the results of these investigations.

This paper is organized as follows. Section II gives a brief review of Thomson's MWM for stationary signals. Section III introduces MW-TFA. In Section IV we apply the MW-TFA to EEG signals and present the results. Some of these results have been described briefly elsewhere [4].

\section{THOMSON'S MWM}

The periodogram is the classical spectral estimator for stationary signals. While it is unbiased, it suffers from high variance. Variance may be reduced by segmenting the signal, computing a periodogram of each segment, and then averaging the individual periodograms. This procedure, however, increases the bias of the spectral estimate. When we have enough data, we may obtain the spectral estimate with satisfactory bias and variance. However, when the data are limited, a tradeoff between bias and variance is inevitable.

For short, time-limited signals, Thomson suggested using a different set of windows to compute several periodograms of the entire signal and then averaging the resulting periodograms to construct a spectral estimate [11]. To get an estimate with 

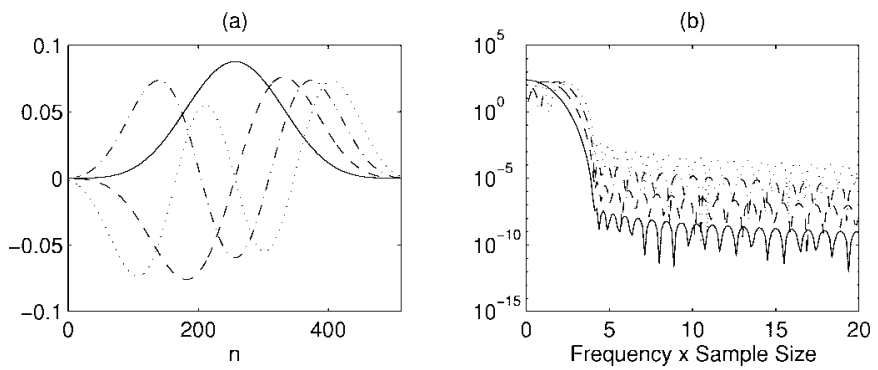

Fig. 1. The first four Slepian sequences and their Fourier transforms for the case $N=512$ and $N W=4$ : (a) the first four individual sequences and (b) complex amplitude square of the Fourier transform of the Slepian sequences in (a). zero-order: solid line; first order: dashed line; second order: dash-dotted line; third-order: dotted line.

low bias and low variance, the windows must be orthogonal (to minimize variance) and optimally concentrated in frequency (to minimize bias). The optimal windows satisfying these requirements for signals with finite length are Slepian sequences or discrete prolate spheroidal sequences.

\section{A. Slepian Sequences}

The Slepian sequences are the eigenvectors of the Toeplitz eigenvalue equation [12]

$$
\sum_{m=0}^{N-1} \frac{\sin 2 \pi W(n-m)}{\pi(n-m)} \nu_{m}^{(k)}(N, W)=\lambda(N, W) \nu_{n}^{(k)}(N, W)
$$

where $N$ is the length of the eigenvectors (or data), and $W$ is a half-bandwidth that defines a small local frequency band centered around frequency $f:\left|f-f^{\prime}\right| \leq W$. Equation (1) is derived from the operation of a time-limitation followed by band-limitation of the signal [12]. Consequently, the Slepian sequences are the orthogonal time-limited functions most concentrated in the frequency band $[-W, W]$.

Slepian sequences are ordered by their eigenvalues. The eigenvalue, $\lambda_{k}$, gives the fraction of energy within the band $[-W, W]$. The first $2 N W$ eigenvalues are very close to 1 , indicating that the spectra of these lower-order Slepian sequences have a large energy concentration within the local frequency band $[-W, W]$. Fig. 1 shows an example of Slepian sequences and their Fourier transform.

\section{B. Brief Description of $M W M$}

The procedure to compute the spectral estimate of $x(n)$, ( $n=0,1, \cdots, N-1$ ) using Thomson's MWM is as follows:

1) Specify $N$ and $W$, where $N$ is the number of data points, and $W$ depends on the desired time-bandwidth $N W$ (or frequency resolution).

2) Use (1) to compute the $\lambda_{k}$ 's and $\nu_{k}$ 's; actually the first $K=2 N W$ terms with the largest eigenvalues are needed. ${ }^{1}$

\footnotetext{
${ }^{1}$ Thomson suggested the use of $K=2 N W-1$ to $K=2 N W-3$ to minimize higher-order window leakage.
}

3) Apply $\nu_{k}$ to the entire length- $N$ data $x(n)$ and take the discrete Fourier transform

$$
x_{k}(f)=\sum_{n=0}^{N-1} x(n) \nu_{n}^{k} e^{-j 2 \pi f n}
$$

where $x_{k}(f)$ is called the $k$ th eigencoefficient and $\left|x_{k}(f)\right|^{2}$ the $k$ th eigenspectrum.

4) Average the $K$ eigenspectra so obtained to get an estimate of the spectrum

$$
\hat{S}(f)=\frac{1}{K} \sum_{k=0}^{K-1} \frac{1}{\lambda_{k}}\left|x_{k}(f)\right|^{2} .
$$

Since the first few eigenvalues are very close to one, (3) can be simplified to

$$
\hat{S}(f)=\frac{1}{K} \sum_{k=0}^{K-1}\left|x_{k}(f)\right|^{2} .
$$

\section{Coherence}

Coherence is a measure of the consistency of phaserelationship between two time series [13]. It provides a frequency-specific measure of the phase coupling between two signals and has been applied to the EEG in a number of clinical and experimental contexts [3], [14].

Coherence can be obtained from one realization of the multichannel data using Thomson's MWM. Given two time series $x(n)$ and $y(n),(n=0,1,2, \cdots, N-1)$, their eigencoefficients $x_{k}(f)$ and $y_{k}(f)$ can be obtained using (2). The estimated coherence is [11]

$$
\gamma_{x y}^{2}(f)=\frac{\left|\sum_{k=0}^{K-1} x_{k}(f) y_{k}^{*}(f)\right|^{2}}{\sum_{k=0}^{K-1}\left|x_{k}(f)\right|^{2} \sum_{k=0}^{K-1}\left|y_{k}(f)\right|^{2}}
$$

where $K \leq 2 N W$ is the number of windows used and the asterisk denotes complex conjugation.

\section{Multiple Window Estimate OF TIME-FREQUENCY DISTRIBUTION}

Thomson's MWM has been extended to TFA [9], [10]. The MW-TFA is applied to the signal in a similar manner as the spectrogram. However, instead of applying a single sliding window along the signal, the MW-TFA applies a set of sliding windows and then takes the average

$$
X_{\mathrm{MW}}(t, f)=\frac{1}{K} \sum_{k=0}^{K-1}\left|X_{k}(t, f)\right|^{2}
$$

where

$$
X_{k}(t, f)=\int x(\tau) h_{k}(\tau-t) e^{-j 2 \pi f \tau} d \tau
$$

$x(t)$ is the signal to be analyzed. 

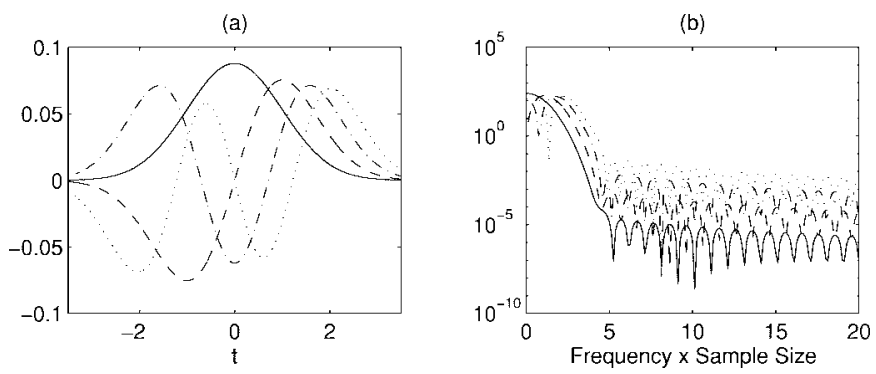

Fig. 2. The first four Hermite functions and their Fourier transforms: (a) first four Hermite functions and (b) complex amplitude square of the Fourier transform of the Hermite functions in (a). Zero-order: solid line; first order: dashed line; second order: dash-dotted line; third order: dotted line.

The MWTF coherence is defined by

$$
\Gamma_{\mathrm{MW}}(t, f)=\frac{\left|\sum_{k=0}^{K-1} X_{k}(t, f) Y_{k}^{*}(t, f)\right|^{2}}{\sum_{k=0}^{K-1}\left|X_{k}(t, f)\right|^{2} \sum_{k=1}^{K-1}\left|Y_{k}(t, f)\right|^{2}} .
$$

Two sets of windows have been used in MW-TFA [9], [10]. One of them is the Slepian sequences described previously and the other is the Hermite functions. We will give a brief overview of Hermite functions before we apply MW-TFA to EEG data and then evaluate these two windowing techniques.

\section{A. Hermite Functions}

Hermite functions are the eigenfunctions of a localization operator over the region [15]

$$
\left\{(t, f): t^{2}+f^{2} \leq R^{2}\right\}
$$

The $k$ th-order Hermite function is defined by

$$
\begin{aligned}
h_{k}(t) & =\pi^{-1 / 4}\left(2^{k} k !\right)^{-1 / 2}\left(t-\frac{d}{d t}\right)^{k} e^{-t^{2} / 2} \\
k & =0,1,2, \cdots
\end{aligned}
$$

Correspondingly, the eigenvalues of the localization operator over the region (9) are given by

$$
\lambda_{k}(R)=1-e^{-R^{2} / 2} \sum_{i=0}^{k} \frac{1}{i !} 2^{-i} R^{2 i} .
$$

An important property of the Hermite functions is that they are optimally concentrated in the circular TF region defined in (9). In a manner similar to Slepian sequences, the eigenvalues $\lambda_{k}$. of the Hermite functions give the fraction of energy within this region. As the $k$ th eigenvalue approaches one, better concentration of the $k$ th order Hermite function occurs. Hence, for a given $R$, there are only a few Hermite functions with good concentration that fall in the region of (9). The first four Hermite functions and their Fourier transforms are shown on Fig. 2.
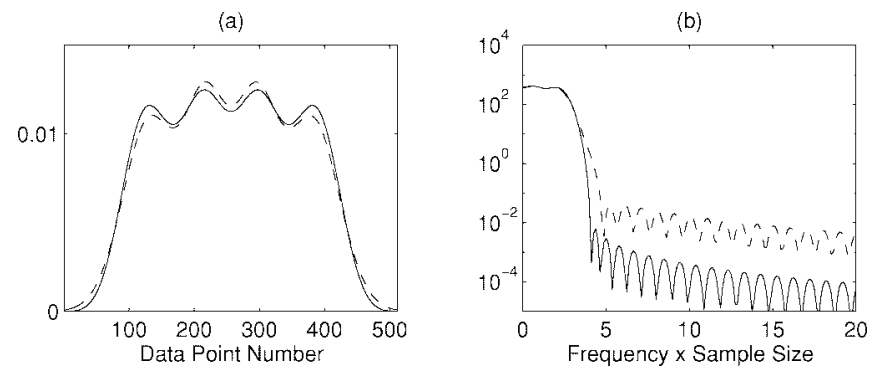

Fig. 3. Sum of the Slepian sequences (solid) and Hermite functions (dashed) in Figs. 1 and 2 in time and frequency domain: (a) sum of the magnitude square and (b) sum of the complex magnitude square of their Fourier transforms. Note the broader bandwidth and higher leakage of Hermite windows.

\section{THE MW-TFA OF EEG}

EEG signals from a previous experiment [4] were used. Thirty seconds of EEG data were recorded simultaneously from the entorhinal cortex and hippocampus of the rat. During the recording, low-intensity trains of electrical pulses were applied to the olfactory cortex. Two type of trains were delivered: 1) trains of pulses ramped from $1-40 \mathrm{~Hz}$ applied in the middle $27 \mathrm{~s}$ and 2) trains of pulses at frequencies between $1-35 \mathrm{~Hz}$ applied in the middle third of the 30-s EEG. In the latter method, the stimulation frequencies remained constant throughout each train.

Time-Frequency Analysis: Both the spectrogram and MWTFA were used in the analysis. The TF distribution was obtained by sliding 2.0-s window(s) with a 1.9-s overlap between consecutive computations. The Hanning window was used for the spectrogram.

Window Matching: The shapes of the Slepian sequences and Hermite functions are very similar (Figs. 1 and 2). To make a fair comparison between the two window functions, the zero-order windows of the two functions were matched with least-mean-square error in the time-domain. The Slepian sequence in Fig. 1 and Hermite functions in Fig. 2 are the matching windows (also see Fig. 3).

\section{A. Results}

1) Time-Frequency Distribution of EEG: Typical EEG samples and their TF distributions are shown in Figs. 4 and 5. The spectrogram (B in Figs. 4 and 5) and MW-TFA images (C, D in Figs. 4 and 5) showed similar features of the EEG signals. Power in the spontaneous EEG was concentrated at low frequencies (under $20 \mathrm{~Hz}$ ). Theta frequency $(4-12 \mathrm{~Hz})$ activity was evident in both the entorhinal cortex and hippocampus EEG recordings (Fig. 5). This is consistent with normal patterns of EEG activity in these sites. Variations in amplitude at frequencies near $7 \mathrm{~Hz}$ in both the entorhinal cortex and hippocampal TF images reflect nonstationarities in theta activity (Fig. 5). Changes in EEG activity induced by the low-intensity stimulation were often more apparent in the TF images than in the time-domain counterparts (Figs. 4 and 5). Power were increased at the stimulation frequency and its upper harmonics (Figs. 4 and 5). The amplitude of train-induced spectral peaks changed with the frequency of stimulation. The peak value was found to be 

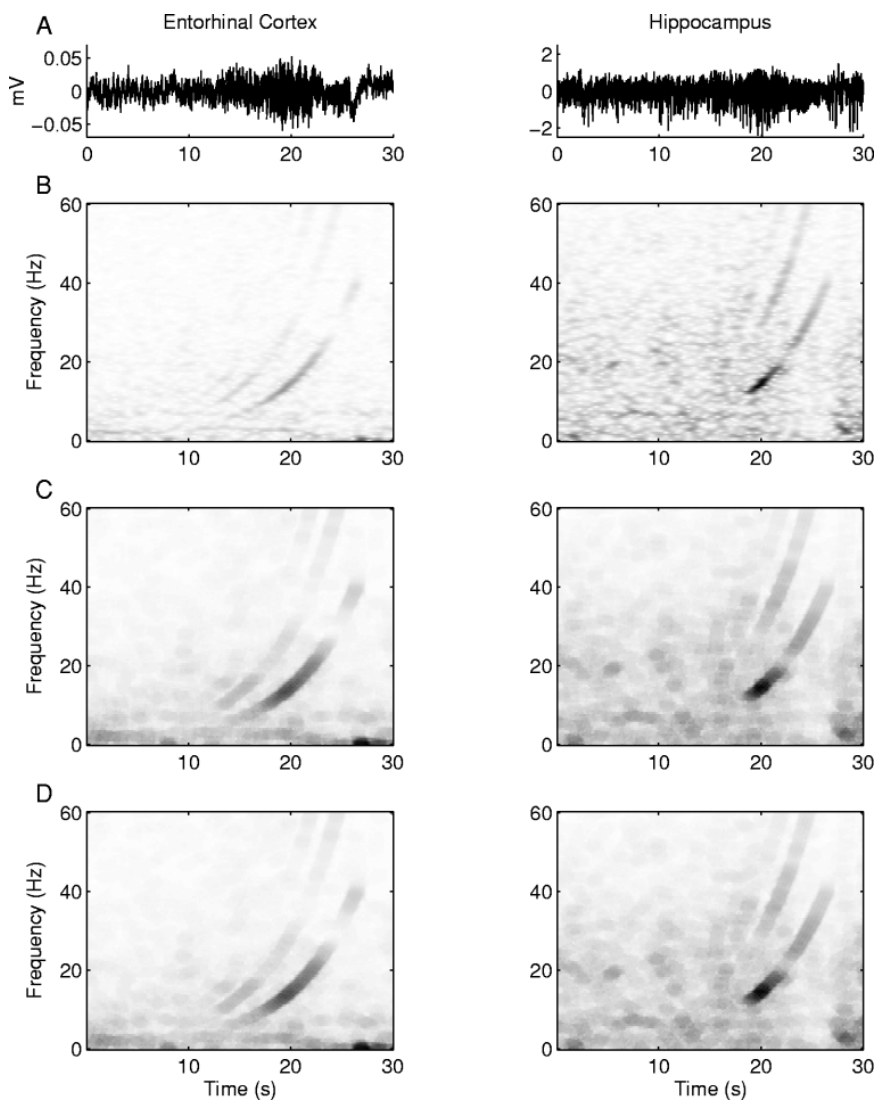

Fig. 4. (A) Examples of raw EEG activity in the entorhinal cortex (left) and hippocampus (right) during stimulation of the olfactory cortex with a pulse-train which ramped from $1-40 \mathrm{~Hz}$. The lower panels show TF image of these EEG using different TF methods. (B) Spectrogram. (C) MW-TFA using Slepian sequences in Fig. 1. (D) MW-TFA using Hermite functions in Fig. 2 The degrees of freedom (DOF) per segment of estimates is approximately two for spectrogram and eight for MW-TFA's. Note all the TFA's show that stimulation-induced responses peaked near $17 \mathrm{~Hz}$ in this animal.

near $15 \mathrm{~Hz}$. A typical example shown in Fig. 4 peaked near $17 \mathrm{~Hz}$.

The differences of the spectrogram and MW-TFA images are also apparent. The MW-TFA results in poor resolution (higher bias) in both time and frequency due to a wider effective time window and broader frequency bandwidth. The variance of MW-TFA representation is lower than that of spectrogram, this being due to the benefit gained from averaging. The resulting MW-TFA representation is more smoothed (lower variance).

2) Time-Frequency Coherence: One advantage of the MWTFA is the ability to obtain TF coherence from one realization of the data, which is impossible with the spectrogram using a single sliding window. The TF coherence in Fig. 6(a) and (b) shows the clear nonstationarity of the coherent activity between the entorhinal cortex and hippocampus in the theta range (near $7 \mathrm{~Hz}$ ).

For comparison, Fig. 6(c) shows TF coherence function calculated (method see [4]) using 10 realizations of EEG recorded during the same stimulation condition. It provided a good coherence estimation for stimulation-induced responses in TF plane. However, the nonstationarity in spontaneous EEG (e.g., theta activity) corresponding to individual realization is not preserved.
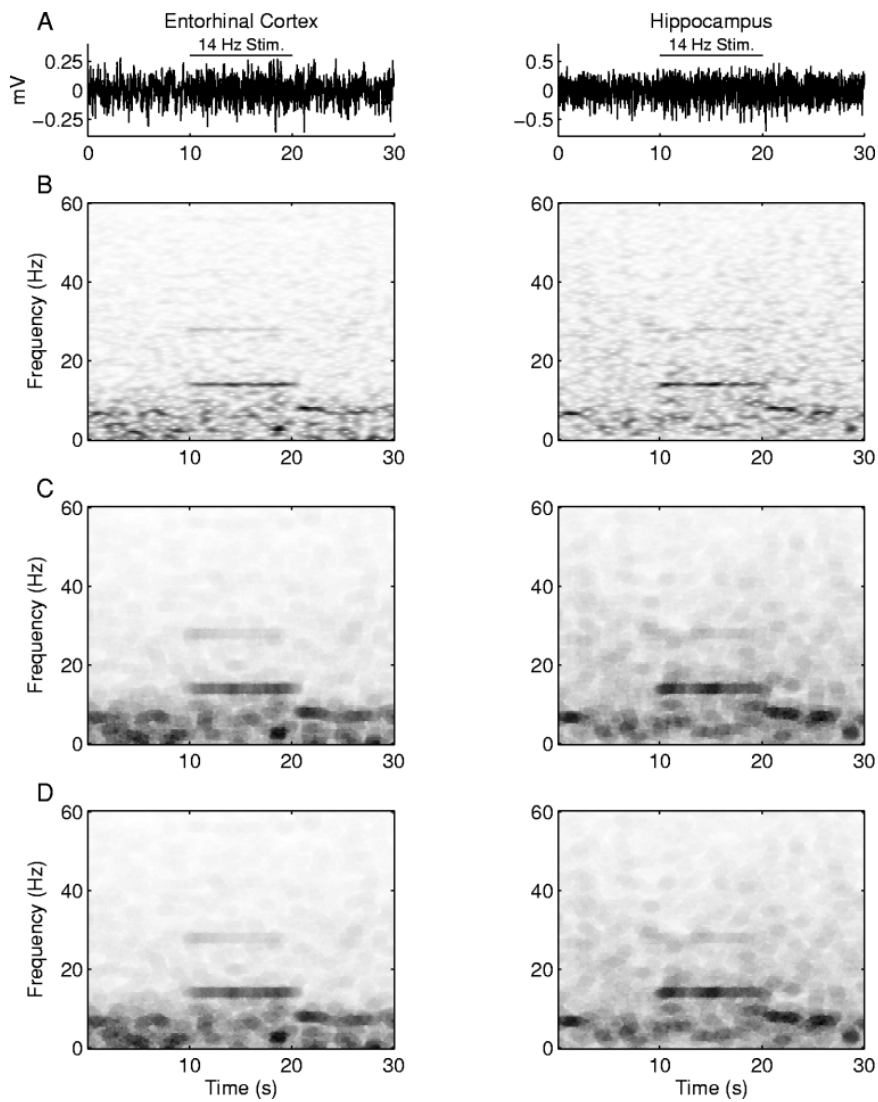

Fig. 5. (A) Examples of raw EEG activity in the entorhinal cortex (left) and hippocampus (right) during application of a low intensity, $14-\mathrm{Hz}$ stimulation train to the olfactory cortex. The lower panels show TF image of these EEG's using different TF methods. (B) Spectrogram. (C) MW-TFA using Slepian sequences in Fig. 1. (D) MW-TFA using Hermite functions in Fig. 2. Note variations in power near $7 \mathrm{~Hz}$ (within the theta band) throughout the sweep, and increased power at $14-\mathrm{Hz}$ input frequency and its harmonics during the middle $10 \mathrm{~s}$.

3) Comparison Between the Two Window Functions: The well-matched multiple windows appear to differ only slightly in the time-domain as shown in Figs. 1-3 for the first four windows. The Hermite windows had higher leakage and wider bandwidth than those of Slepian sequences (Fig. 3) in the frequency domain. Both the higher leakage and broader bandwidth will introduce more bias into the estimation. However, the differences of the two functions did not introduce a noticeable difference in the MW-TFA results, as can be seen from Figs. 4-6.

\section{DISCUSSION}

The MW-TFA of EEG showed similar results as spectrogram. These results indicate that signal transmission from the piriform cortex via entorhinal cortex to the hippocampus is optimal for frequencies of neural activity near $15 \mathrm{~Hz}$. It has been reported recently that odors from predator triggers responses in this frequency range in the olfactory cortex and hippocampus [16]. This finding, together with our results, suggests that frequency near $15 \mathrm{~Hz}$ effectively transmit EEG activity between sites in the olfactory system and points to a role that oscillatory states play in determining the flow of neural activity from one brain site to another [16]. 
(a)

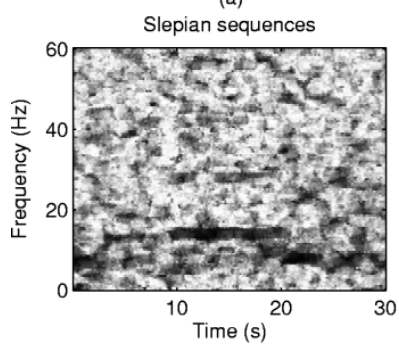

(b)

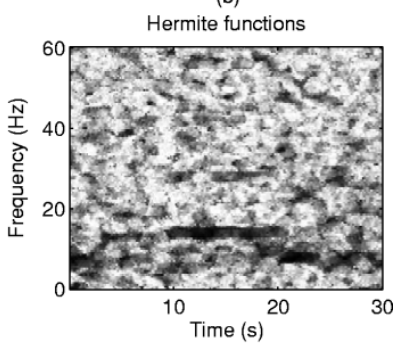

(c)

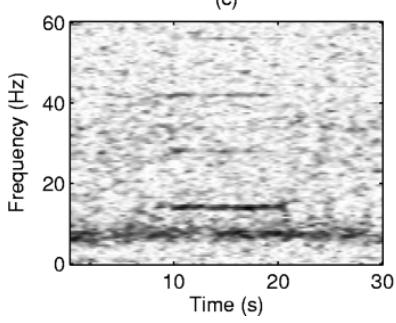

MWM to estimate the spectrum at time $t$. Hence, it is still a problem of spectral estimation of the time-limited signal. Since the Slepian sequences are optimal for the analysis of time-limited signals, for matching windows in time domain for Slepian sequences and Hermite functions, the estimation using Slepian sequences should give better estimates. The Hermite functions and associated eigenvalues, however, are much easier to calculate in practice. If varying windows are necessary to capture time-varying components in the signal, the simplicity of calculation of the Hermite functions might outweigh the loss of optimality.

\section{REFERENCES}

[1] H. Berger, "Uber das elektrenkephalogramm des menschen," Archiv fur Psychiatrie und Nervenkrankheiten, vol. 87, pp. 527-570, 1929.

[2] W. Scheuler, P. Rappelsberger, F. Schmatz, C. Pastelak-Price, H. Petsche, and S. Kubicki, "Periodicity analysis of sleep EEG in the second and minute ranges-Example of application in different alpha activities in sleep," Electroencephalogr. Clin. Neurophysiol., vol. 76, pp. 222-234, 1990.

Fig. 6. (a) and (b) MW TF coherence functions for the entorhinal cortex and hippocampus data shown in Fig. 5. Note the enhanced coherence at the 14-Hz input frequency and its upper harmonics during the middle $10 \mathrm{~s}$. Also note high coherence values in theta range (near $7 \mathrm{~Hz}$ ) only at times (at the beginning of first and third $10 \mathrm{~s}$ ) when theta activity appears in both the entorhinal cortex and hippocampus. (c) TF coherence functions calculated using multiple samples of EEG. Ten realizations of EEG recorded in the same stimulation condition $(14 \mathrm{~Hz})$ from the same animal were used. Although it shows high coherence values in the theta range (near $7 \mathrm{~Hz}$ ), the nonstationarity of theta activity corresponding to each realization was not preserved.

The MW-TFA has been demonstrated as a useful tool to estimate the TF distribution. Its strengths lie in providing a low bias and low variance estimate for a single realization of data, which is usually the case for nonstationary timevarying signals. Although the MW-TFA has poorer time and frequency resolutions than those of the spectrogram, it has a lower variance. This is consistent with the observation made by Frazer and Boashash [9]. The most appealing property of the MW-TFA is that it enables us to estimate the TF coherence from a single realization of the time series. This provides us an effective tool for investigating the temporal relationship between EEG signals in different brain regions from time to time. To our knowledge, the work reported in this paper is the first attempt to extend Thomson's MW coherence to TF coherence.

We have made a fair comparison between the Slepian sequences and Hermite functions by matching them in terms of producing least-mean-square error in the time-domain. Although there were slight differences in the time- and frequency-domains for the first few windows, there were no apparent differences in the TF representations.

The Slepian sequences can also be viewed as the eigenfunctions of a localization operator over a rectangular region- $[-T, T] \times[-W, W]$ in the $\mathrm{TF}$ plane [15]. This rectangular region is maintained when $2 N W$ windows are all employed. When we use the first few windows, these windows concentrate in a similar circular region in $\mathrm{TF}$ plane as that of Hermite functions.

In the way the MW-TFA is implemented, it is the natural extension of Thomson's MWM. The MW-TFA performs multiple window spectral estimation in a piecewise fashion. Within a given window centered in time $t$, it uses the Thomson's
[3] P. H. Boeijinga and F. H. Lopes da Silva, "Modulations of EEG activity in the entorhinal cortex and forebrain olfactory areas during odour sampling," Brain Res., vol. 478, pp. 257-268, 1989.

[4] S. Haykin, R. J. Racine, Y. Xu, and C. A. Chapman, "Monitoring neuronal oscillations and signal transmission between cortical regions using time-frequency analysis of electroencephalographic activity," Proc. IEEE, vol. 84, pp. 1295-1301, Sept. 1996.

[5] H. Sugimoto, N. Ishii, A. Iwata, N. Suzumura, and T. Tomita, "On the stationarity and normality of the electroencephalographic data during sleep stages," Comput. Prog. Biomed., vol. 8, pp. 224-234, 1978.

[6] E. A. Bartnik and K. J. Blinowska, "Wavelets-new method of evoked potential analysis," Med. Biol. Eng. Comput., vol. 30, pp. 125-126, 1992.

[7] Z. Lin and J. D. Z. Chen, "Advances in time-frequency analysis of biomedical signals," Crit. Rev. Biomed. Eng., vol. 24, pp. 1-72, 1996.

[8] W. Martin and P. Flandrin, "Wigner-Ville spectral analysis of nonstationary process," IEEE Trans. Acoust., Speech, Signal Processing, vol. 33 , pp. 1461-1470, 1985.

[9] G. Frazer and B. Boashash, "Multiple window spectrogram and timefrequency distributions," in Proc. IEEE Int. Conf. Acoustic, Speech, and Signal Processing-ICASSP'94, 1994, vol. IV, pp. 293-296.

[10] M. Bayram, "Multiple window time-frequency analysis," M.S. thesis, Department of Electrical and Computer Engineering, Rice University, Houston, TX, 1996.

[11] D. J. Thomson, "Spectrum estimation and harmonic analysis," Proc. IEEE, vol. 70, pp. 1055-1096, 1982.

[12] D. Slepian, "Prolate spheroidal wave functions, Fourier analysis, and uncertainty-V: The discrete case," Bell Syst. Tech. J., vol. 57, pp. 1371-1430, 1978.

[13] S. L. Marple, Digital Spectral Analysis with Applications. Englewood Cliffs, NJ: Prentice-Hall, 1987.

[14] J. C. Shaw, "Correlation and coherence analysis of the EEG: A selective tutorial review," Int. J. Psychophysiol., vol. 1, pp. 255-266, 1984.

[15] I. Daubechies, "Time-frequency localization operators: A geometric phase space approach" IEEE Trans. Inform. Theory, vol. IT-34, pp.

[16] V. R. Heale, C. H. Vanderwolf, and M. Kavaliers, "Components of weasel and fox odors elicit fast wave bursts in the dentate gyrus," Behavioral Brain Res., vol. 63, pp. 159-165, 1994.

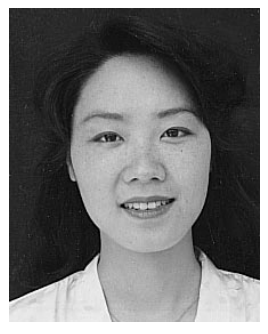

Yan Xu received the B.Sc. degree in biomedical engineering and instrumentation from Zhejiang University, Zhejiang, PR China, in 1987, and the M.Sc. degree in neuroscience from Queen's University, Kingston, Ont., Canada, in 1993.

She was a Research Fellow in the Department of Psychology and Communications Research Laboratory at McMaster University, Hamilton, Ont Canada, from 1993 to 1997. She is currently with IBM, Binghamton, NY. Her research interests include biosignal processing and neural networks. 605-612, 1988. 


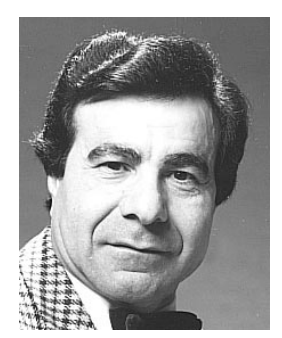

Simon Haykin (SM'70-F'82) received the B.Sc. degree (first class honors) in 1953, the Ph.D. degree in 1956, and the D.Sc. degree in 1967, all in electrical engineering from the University of Birmingham, Birmingham, U.K

$\mathrm{He}$ is the founding Director of the Communication Research Laboratory at McMaster University, Hamilton, Ont., Canada. His research interests include nonlinear dynamics, neural networks, adaptive filters, and their applications in radar and communication systems. In 1996, he was awarded the title of University Professor. He is the Editor for the series Adaptive and Learning Systems for Signal Processing, Communications and Control (New York: Wiley-Interscience)

In 1980, Dr. Haykin was elected a Fellow of the Royal Society of Canada. $\mathrm{He}$ is a recipient of the Canadian Telecommunications Award from Queen's University.

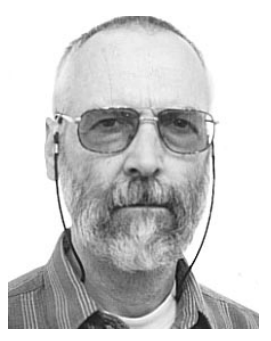

Ronald J. Racine received the B.Sc. degree in psychology from the University of Oregon, Eugene, in 1965 and the Ph.D. degree in psychology from McGill University, Montreal, P.Q., Canada, in 1969.

He is currently a Professor in the Department of Psychology, McMaster University, Hamilton, Ont Canada. His research interests include the physiology of learning and memory and the physiology of epilepsy. 